\title{
Klassifikation von Azetabulumfrakturen
}

\author{
Manfred Römer, Bernd Wittner
}

\section{Zusammenfassung}

Die Frakturmorphologie der Azetabulumfrakturen ist sehr vielfältig, entsprechend komplex ist die Klassifikation dieser Frakturen. Die heute am häufigsten gebrauchte Einteilung ist die Klassifikation der Azetabulumfrakturen, die von der AO erarbeitet wurde. Sie beruht auf der Klassifikation von Judet und Letournel. Während

\section{Einleitung}

Die operativen Versorgungsmögichkeiten von Azetabulumfrakturen haben sich in den letzten Jahren erheblich verbessert, und um Frakturen und Ergebnisse vergleichen zu können wurden Einteilungen und Klassifikationen geschaffen, die den chirurgischen Bedürfnissen gerecht werden.

So wurde in den ersten Klassifikationen lediglich die Stellung des Hüftkopfes zum frakturierten Azetabulum beschrieben, was zur Bewertung der oft sehr komplexen Brüche und zur operativen Entscheidung wenig hilfreich ist (Tab.1).

1964 beschrieben die Pioniere der Azetabulumchirurgie Judet und Letournel eine verbesserte Einteilung der Azetabulumfrakturen, die sie nach Dokumentationen und Nachkontrollen einer großen Anzahl operierter Azetabulumfrakturen erarbeiteten und die auch heutigen Ansprüchen noch gerecht wird. Diese Einteilung wurde später durch die detaillierte AO-Klassifikation ergänzt bzw. in diese integriert.

OP-JOURNAL 2003; 19: 151-155

(c) Georg Thieme Verlag Stuttgart . New York letztere jedoch lediglich die Frakturformen beschreibt, ordnet die AO Klassifikation die Frakturtypen nach Ihrer Prognose und der Schwierigkeit der operativen Rekonstruktion. Im klinischen Alltag wird die Klassifikation bis zu den Frakturgruppen verwendet, während für wissenschaftliche Auswertungen meist die komplette Einteilung verwendet wird. det und ist hier in dem Zwickel eines umgekehrten, bildlich betrachteten Ypsilons, das die Tragpfeiler im Beckenknochen versinnbildlicht, verankert.

Grundlage der Einteilung der Azetabulumfrakturen nach Judet und Letournel ist die anatomische „Pfeilertheorie“.

Azetabulumfrakturen entstehen durch massive direkte oder indirekte Krafteinwirkungen in das Gelenk, entsprechend hoch sind Begleitverletzungen an anderen Skelettabschnitten und inneren Organen, bes. im Beckenbereich (siehe hierzu Artikel 15 dieser Ausgabe).
Tab. 1 Frühere Klassifikationen der Azetabulumfrakturen

1951 - Klassifikation nach Cauchoix/Tuchet 1954 Böhler

$1961-$ Creyssel/Schnepp

\section{Einteilung nach Judet und Letournel}

Die Grundlage der Letournel'schen Einteilung ist die anatomische Pfeilertheorie. Das Azetabulum wird aus dem Os ilium, Os ischiadicum und dem Os pubis gebil-
Entscheidend für die Frakturform ist die Stellung des Hüftkopfes zum Azetabulum im Moment der Krafteinteilung, sowie auch individuelle Faktoren wie z. B. Osteoporose beim alten Menschen.

Mitentscheidend für die Frakturform ist neben der Richtung der Krafteinleitung die Stellung des Hüftkopfes zum Azetabulum

Zur radiologischen Beschreibung des Frakturverlaufes werden als Minimum
Tab. 2 Einteilung nach Judet und Letournel

Grundtypen:

Typ 1 - Fraktur hinterer Pfannenrand/mit und ohne Luxation des Hüftkopfes nach dorsal

Typ 2 - Abrissfraktur dorsaler Pfeiler mit Dislokation, oft dorsmed. Hüftkopfsub-/-luxation

Typ 3 - Abbruch des ventralen Pfannenrandes

Typ 4 - Abbruch des ventralen Pfeilers mit Dislokation, ventromed. Hüftkopfsub-/-luxation Typ 5 - Querfrakturen durch das Acetabulum, Pfannendach intakt

(Abb. 1a-e)

Kombinationsverletzungen:

Typ 6 - T-Frakturen, Hüftkopf medialisiert

Typ 7 - Fraktur hinterer Pfeiler mit hinterem Pfannenrand

Typ 8 - Querfraktur mit hinterem Pfannenrandbruch

Typ 9 - Abriss des ventralen Pfeilers mit hinterem Querbruch

Typ 10 - Zweipfeilerfraktur

(Abb. 2a-e) 

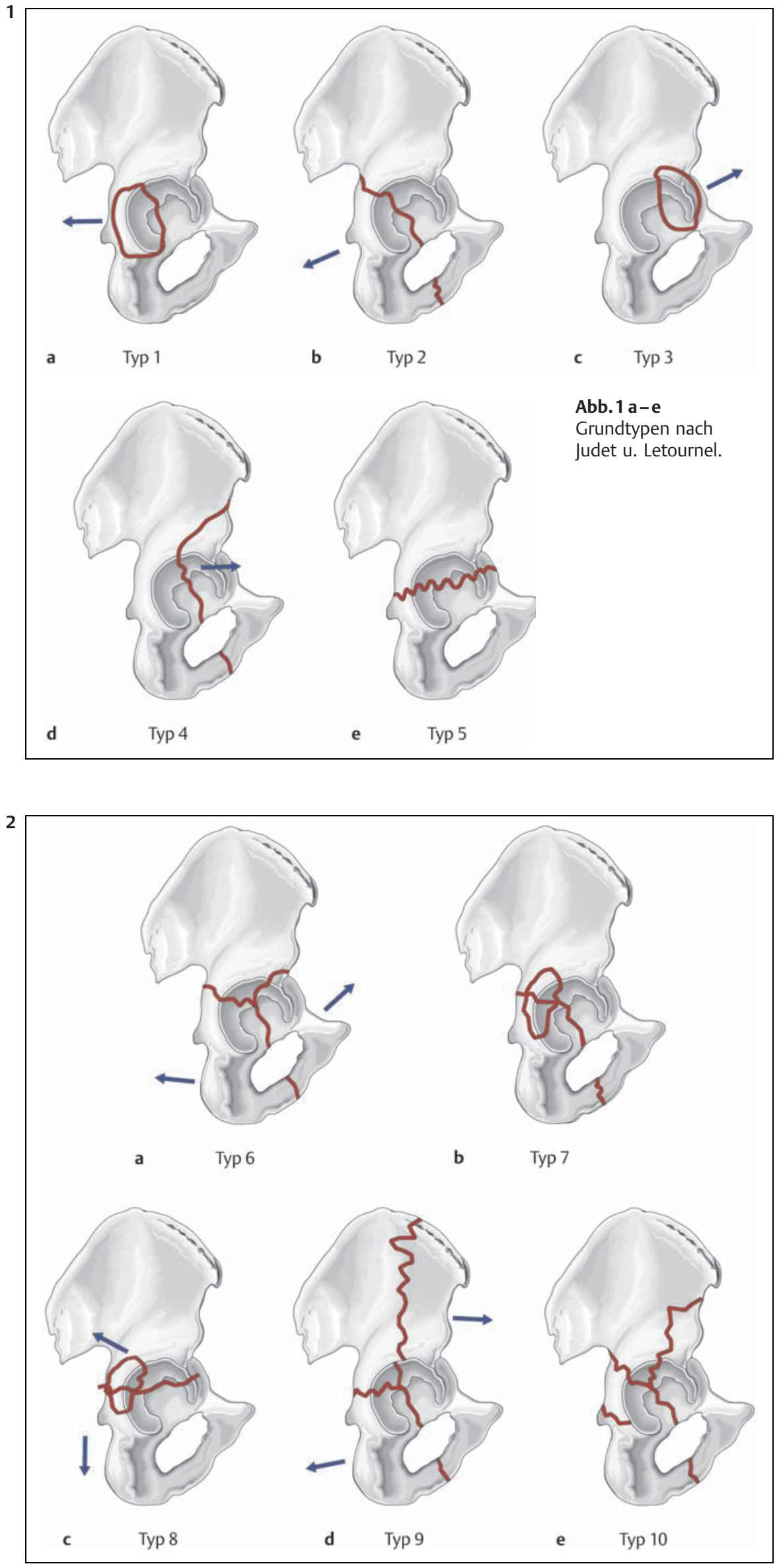

folgende Röntgenbildeinstellungen benötigt (s. auch Artikel 11 dieses Heftes):

Beckenübersicht

Alaaufnahme zur Beurteilung des hinteren Pfeilers

Obturatoraufnahme zur Beurteilung des vorderen Pfeilers

Wobei heute eine zusätzliche CT-Untersuchung ohne, oder mit 3-D-Rekonstruktion bei komplexeren Verletzungen fast immer angefertigt werden sollte.

Radiologische Minimaldiagnostik zur Beurteilung von Azetabulumfrakturen: Beckenübersicht, Ala- und Obturatoraufnahme

Die Einteilung von Letournel ist relativ komplex. Sie unterscheidet 2 Haupttypen: elementare Frakturen und komplexe Frakturen, die sich aus Kombinationen der Elementarfrakturen ergeben. Er beschreibt fünf elementare Frakturen (Grundtypen) sowie weitere 5 Komplexe Frakturen (Kombinationstypen) (s. Tab. 2).

In der Klinik sind der hintere Pfannenrandbruch (Typ 1) und die Kombinationsverletzungen mit Bruch beider Pfeiler (Typ 10) bzw. Querbruch des Azetabulums mit hinterem Pfannenrandbruch (Typ 8) am häufigsten zu finden, während der vordere Pfannenrandbruch (Typ 3) nur sehr selten auftritt (s. Tab. 3)

Tab. 3 Frakturhäufigkeiten nach Letournel

\begin{tabular}{|c|c|}
\hline vorderer Pfannenrandbruch (Typ 3) & $1,9 \%$ \\
\hline hinterer Pfeilerbruch (Тyp 2) & $3,2 \%$ \\
\hline $\begin{array}{l}\text { hinterer Pfeiler- und Pfannenrand- } \\
\text { bruch (Typ 7) }\end{array}$ & \\
\hline vorderer Pfeilerbruch (Typ 4) & $\%$ \\
\hline $\begin{array}{l}\text { vorderer Pfeiler- und hinterer } \\
\text { Hemiquerbruch (Typ 9) }\end{array}$ & \\
\hline T-Bruch (Typ 6) & $7,0 \%$ \\
\hline reiner Querbruch (Typ 5) & $7,4 \%$ \\
\hline $\begin{array}{l}\text { Quer- und hinterer Pfannenrand- } \\
\text { bruch (Typ 8) }\end{array}$ & $19,6 \%$ \\
\hline Bruch beider Pfeiler (Typ 10) & \\
\hline hinterer Pfannenrandbruch (Typ 1) & 23 \\
\hline
\end{tabular}

Abb. 2a-e Kombinierte Typen nach Judet und Letournel. 
Die dorsalen Pfannenrandbrüche (Typ1), die Brüche beider Pfeiler (Typ 10) und der Querbrüche des Azetabulums mit hinterem Pfannenrandbruch (Typ 8) sind die häufigsten Frakturformen am Azetabulum

Prinzipiell kann die Zuordnung der Frakturen zu den Typen anhand der Beckenübersichtsaufnahme sowie zwei Schrägaufnahmen (Ala- und Obturatoraufnahme) erfolgen, wobei sich wegen der komplexen Anatomie heute die Durchführung eines CT mit 3D-Rekonstruktion anbietet (s. Artikel Nr. 11 in diesem Heft).

\section{Klassifikation der AO}

Die Klassifikation von Judet und Letournel beschreibt die Frakturen des Azetabulums rein anatomisch, ohne dass dabei wichtige prognostische Faktoren berücksichtigt werden wie z.B. ob der kraniale Gewicht-tragende Gelenkanteil intakt ist oder wie ausgeprägt die Dislokation der Fragmente ist, bzw. in welche Richtung die Fragmente disloziert sind. Letzteres ist aber für die Wahl des operativen Zugangs mit entscheidend. Zusätzlich wird die hintere Dislokation des Hüftkopfes nur ungenügend berücksichtigt. Sie stellt aber einen wichtigen prognostischen Faktor in Bezug auf die Mitverletzung des Nervus ischiadicus und das Auftreten einer Hüftkopfnekrose im weiteren Verlauf dar. Deshalb wurde die Klassfikation von Judet und Letournel von der AO weiterentwickelt.

Entsprechend dem Schema zur Klassifikation der langen Röhrenknochen erfolgt die Einteilung in 3 Typen $(A B C)$ mit je drei Gruppen (A.1; A.2...C.2, C.3) die jeweils wieder in drei Untergruppen eingeteilt werden (A.1.1, A.1.2 . .C.3.2, C.3.3). Wobei der Schweregrad der Verletzung innerhalb der Typen, Gruppen und Subgruppen zunimmt. Das heißt, die Prognose der Verletzung wird bei adäquater Behandlung ernster.

Die Prognose einer Fraktur wenn sie adäquat behandelt wird, ist besser je weiter „vorne“ in der Klassifikation die Frakturform aufgeführt wird.

Der Typ A beschreibt partielle Gelenkfrakturen, bei denen der Hauptteil des Gelenks intakt geblieben ist.

Abb.3a-j Typen und Gruppen der Azetabulumfrakturen nach der AO-Einteilung.
Tab. 4 Einteilung der AO

Typ A - partiell artikulär, eine der beiden Pfeiler/Säulen ist betroffen (Abb.4a-c)

A1 - Fraktur hinterer Pfannenrand, vielgestaltig

A2 - Fraktur hinterer Pfeiler

A3 - Fraktur vorderer Pfannenrand oder des vorderen Pfeilers

Typ B - partiell artikulär, mit Querbruch/T-Fraktur/Pfannendachbeteiligung (Abb.4d-g)

B1 - reiner Querbruch mit und ohne hinterem Pfannenrand

B2 - T-förmiger Frakturverlauf

B3 - Fraktur vorderer Pfeiler oder vorderer Pfannenrand mit hinterem Transversalbruch

Typ C - Fraktur beider Pfeiler, komplett artikulär, alle Fragmente von llium abgelöst (Abb. $4 \mathrm{~h}-\mathrm{j}$ )

C1 - Fraktur vorderer Pfeiler bis Crista iliaca, hohe Variabilität

C2 - Fraktur vorderer Pfeiler bis an die Iliumgrenze, geringe Variabilität

C3 - Frakturen bis in das Iliosakralgelenk hinein

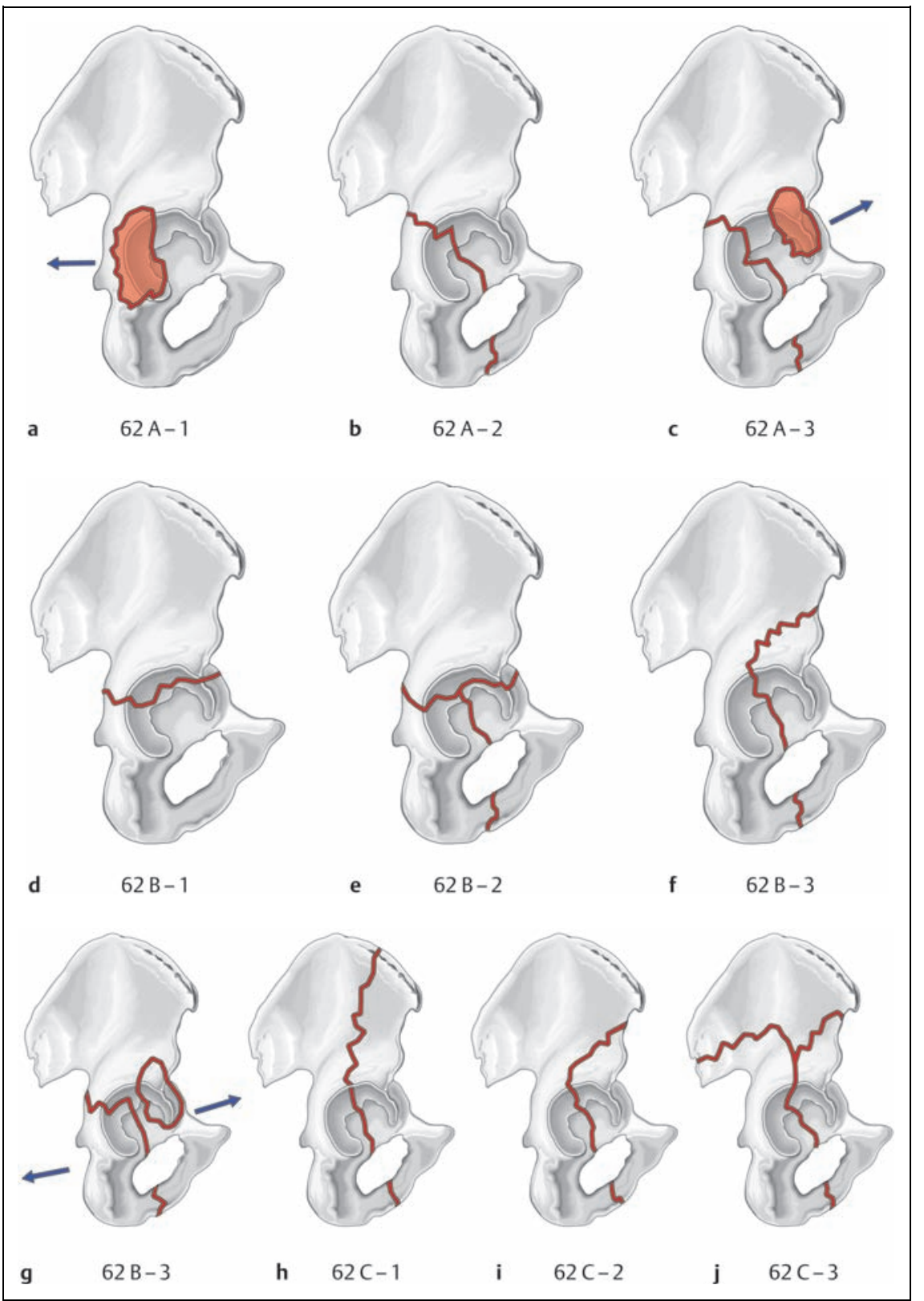


Tab. 5 Subgruppen des Typs A der Azetabulumfrakturen

A.1: Isolierte Fraktur des hinteren Pfannenrandes

A.1.1 einfache Fraktur (ein Fragment)

A.1.2 Mehrfragmentfraktur

A.1.3 Pfannenrandfraktur mit Impression der Gelenkfläche am Azetabulum

A.2: Fraktur des dorsalen Pfeilers

A.2.1: Fraktur isoliert im Sitzbein ohne Beteiligung des Schambeinastes

(entspricht ausgedehnter hinterer Pfannenrandfraktur)

A.2.2: Typische hintere Pfeilerfraktur (von der Inzisura ischiadica durch das Acetabulum in das Foramen obturatorium, unter Mitbeteiligung des Schambeinastes)

A.2.3: Kombination aus hinterem Pfannenrandbruch und hinterem Pfeilerbruch

A.3: Fraktur des vorderen Pfannenrandes und oder Fraktur des vorderen Pfeilers

A.3.1: Isolierte vordere Pfannenrandfraktur (fast immer in Kombination mit einer Luxation des Hüftkopfes nach vorne (seltenster Fraktur-Typ)

A.3.2: Die Frakturlinie durch den vorderen Pfeiler beginnt im Verlauf der Crista iliaca.

A.3.3: Die Frakturlinie durch den vorderen Pfeiler beginnt unterhalb der Spina iliace anterior superior.

Im Typ B sind Querfrakturen und T-Frakturen der Gelenkfläche zusammengefasst, bei denen der wichtige kraniale Gelenkanteil über einen intakten Anteil des Ileums mit dem Achsenskelett in Verbindung geblieben ist.

Während bei den C-Frakturen das gesamte Azetabulum aus dem Skelett herausgebrochen ist (sog. „Floating Acetabulum“) s. Tab. 4 und Abb. $3 \mathbf{a}-\mathbf{j}$.

Die weitere Unterteilung der Gruppen in Subgruppen soll hier nur exemplarisch am Typ A aufgezeigt werden (Tab.5):

Die Gruppen der A1-Frakturen ist die häufigste Frakturgruppe bei den Azetabulumfrakturen. Entsprechend ihrer Stellung in der Klassifikation gelten Sie als die „gutartigsten“ Brüche. Entsprechend den Grundlagen des AO-Klassifizierungs-

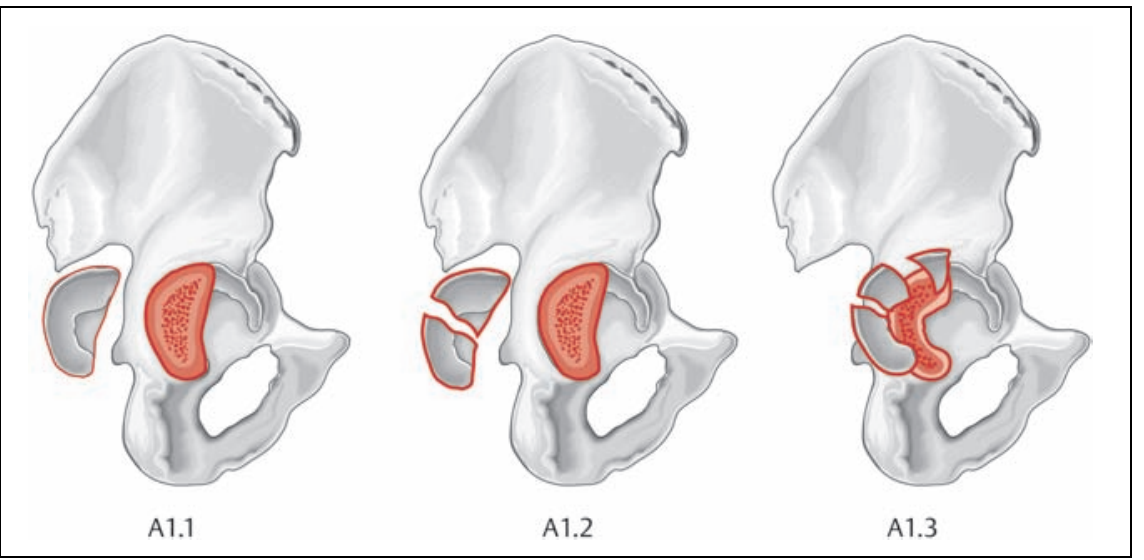

Abb.4 Subgruppen der Gruppe A1 (Frakturen des dorsalen Pfannenrandes)

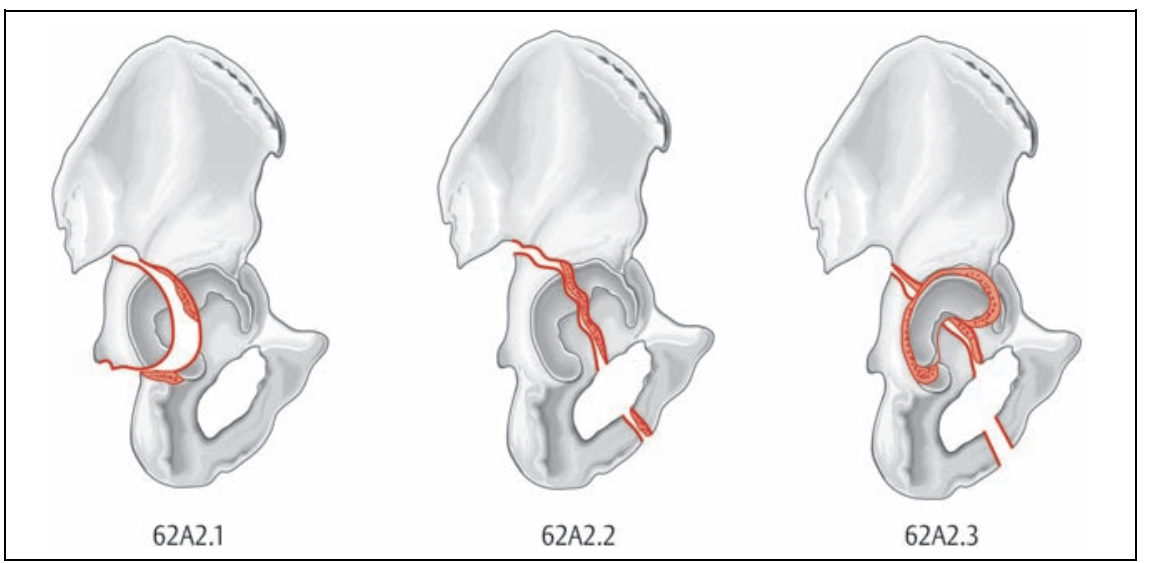

Abb. 5 Subgruppen der Gruppe A2 (Frakturen des dorsalen Pfeilers).

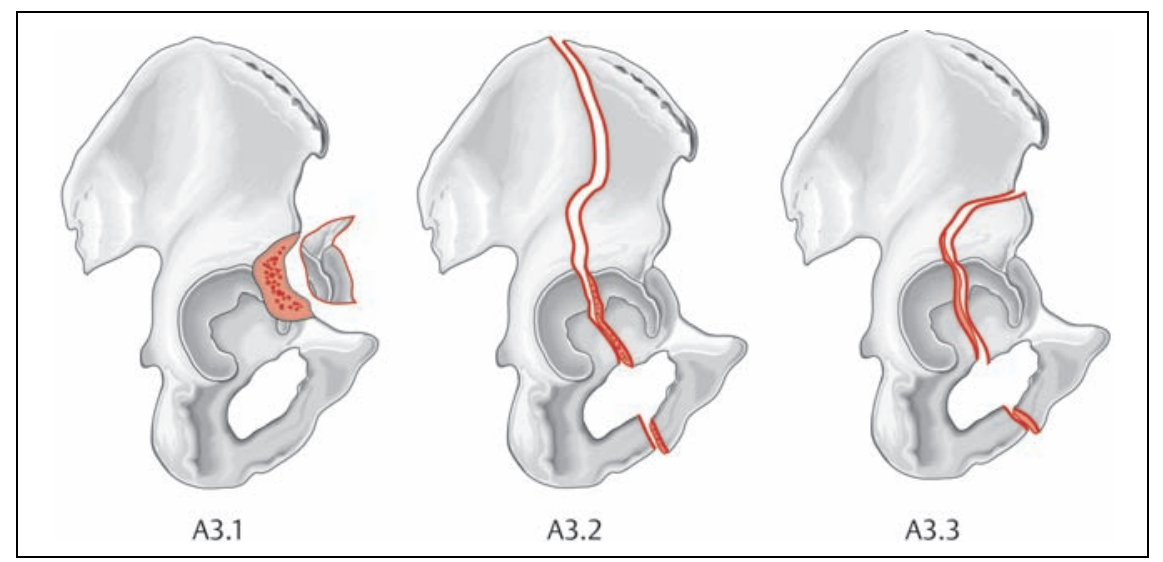

Abb. 6 Subgruppen der Gruppe A3: (Frakturen des ventralen Pfeilers).

systems setzt die Stellung einer Frakturform innerhalb der Klassifizierung voraus, dass die Fraktur „adäquat“ behandelt wird: Die Ergebnisse der Frakturen der Gruppe A1 ist nur dann gut, wenn das Fragment operativ reponiert und stabil fixiert wird. Während die operative Refixierung und Stabilisierung des Fragments zu guten Ergebnissen führt, ist die früher häufig durchgeführte konservative Behandlung des Hüftgelenks mit frühen Arthrosen oder rezidivierenden Verrenkungen verbunden. Wobei die Frakturen der Subgruppe A.1.3 infolge des zu erwartenden Knorpelschadens eine deutlich schlechtere Prognose haben als die Frakturen der Subgruppen A.1.1 und A.1.2 (Abb.4). 


\section{Beispiele von Acetabulumfrakturen}

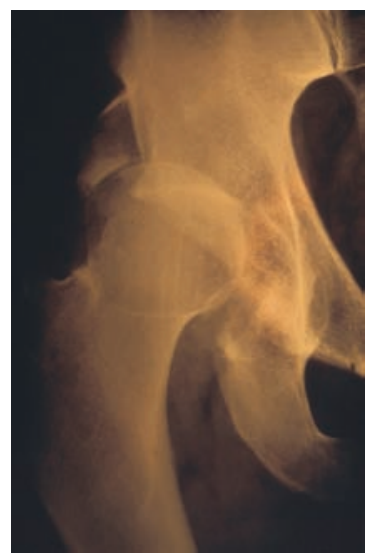

Abb.7 Hintere Pfannenrandfraktur mit einem Abrissfragment (AO-62 A.1; Letournel Typ 1).

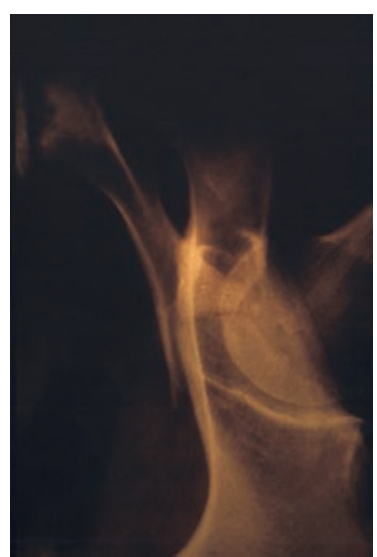

Abb. 9 Vordere Pfeilerfraktur und Querfraktur (AO B.2; Letournel Typ 9).

Die gute Prognose der A.1-Frakturen ist gebunden an eine adäquate Behandlung.

Die Frakturen der Gruppe A2 gehören zu den seltenen Frakturen des Azetabulums (Abb.5).

Die Zuordnung der A.3-Frakturen mit Beginn unterhalb der Spina iliaca und Frakturen durch die Crista iliacain unterschiedliche Subgruppen und deren Reihenfolge innerhalb der Klassifizierung ergibt sich aus der klinischen Erfahrung: Obwohl letztere in stärkerem Ausmaß

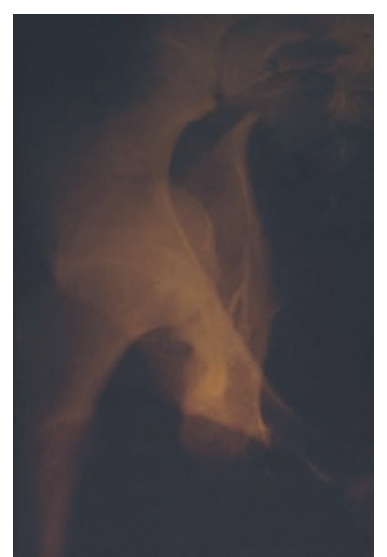

Abb. 8 Abrissfraktur des hinteren Pfeilers (AO A.2; Letournel Typ 2).

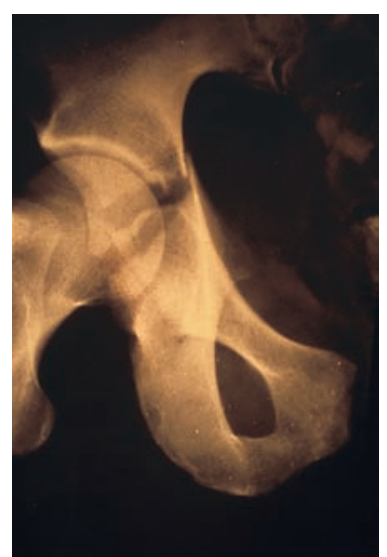

Abb.10 Querfraktur des Acetabulums (AO B.1; Letournel Typ 5).

den Gewichts-tragenden Dom des Azetabulums involvieren, sind letztere meist weniger disloziert und operationstechnisch meist besser anzugehen (s. Artikel 12 dieser Ausgabe) (Abb.6)

Die Zuordnung zu den Gruppen und Subgruppen der AO-Klassifizierung orientiert sich nicht nur am anatomischen Verlauf, sondern an der klinischen Erfahrung mit den Frakturformen.

Die differenzierte Besprechung aller Subgruppen würde den Rahmen dieser Arbeit sprengen, interessierte Leser seien auf die Literaturhinweise verwiesen.
Auch bei der Zuordnung der Frakturformen zu den anderen Subgruppen ist entsprechend der Philosophie der AO-Klassifizierung nicht nur der anatomische Frakturverlauf, sondern auch klinische Erfahrungswerte wie die Häufigkeit von Begleitverletzungen, der zu beobachtende Dislokationsgrad und die Schwierigkeit der operativen Rekonstruktion mitentscheidend.

\section{Schlussfolgerung}

In Deutschland werden zurzeit noch zwei Klassifikationen der Azetabulumfrakturen parallel gebraucht. Während die Einteilung von Letournel und Judet nur die anatomischen Frakturverläufe beschreibt, ist die Grundlage der AO-Klassifizierung die Prognose der einzelnen Frakturformen und die Schwierigkeit bei der operativen Rekonstruktion. Für wissenschaftliche Auswertungen findet die komplette Klassifikation Anwendung, im klinischen Alltag reicht meist die Unterteilung bis zu den Frakturgruppen, wie sie hier beschrieben ist, aus.

\section{Literatur}

1 Letournel E, Judet R. Fractures of the Acetabulum, Springer Verlag 2. Aufl. 1993

2 Tile M. Fractures of the Pelvis an the Acetabulum, Williams \& Wilkins Verlag 2. Aufl. 1995

${ }^{3}$ Helfet DL, Bartlett CS. III Acetabulumfrakturen, Beurteilung, Klassifikation, Zugänge und Behandlungskonzepte. In: T.P. Rüedi, W.M. Murphy AO Prinzipien des Frakturmanagements. Thieme Verlag 2003

4 Bray TJ. Osteosynthese - Arbeitsbuch und Atlas VCH Verlagsgesellschaft Weinheim 1993

5 Kremer, Lierse, Platzer, Schreiber, Weller, Platzer, Trentz et al. Chirurgische Operationslehre Band 8. Azetabulum. Thieme Verlag 1997

\section{Dr. med. Manfred Römer \\ Oberarzt}

\section{Dr. med. Bernd Wittner}

Chefarzt

Unfallchirurgische Abteilung

Hohenloher Krankenhaus GmbH

Stettenstr. 32

D-74653 Künzelsau 